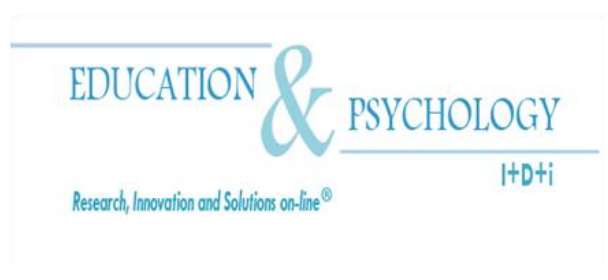

\title{
Relación entre Alta Ideación Suicida y Variables Psicosociales en Estudiantes Universitarios
}

\section{David Sánchez-Teruel, Ana García-León y José A. Muela-Martínez}

Departamento de Psicología de la Facultad de Humanidades y Ciencias de la Educación, Universidad de Jaén, Jaén

\section{España}

Correspondencia: David Sánchez-Teruel Campus las lagunillas, s/n Edificio C5 23071 JAÉN (España) dsteruel@ujaen.es

(C) Education \& Psychology I+D+i and Editorial EOS (Spain) 


\section{Resumen}

Introducción. Los universitarios presentan unas elevadas tasas de ideación suicida asociadas a menudo con diversos factores psicosociales. El objetivo de este estudio fue evaluar si algunas de estas variables psicosociales están relacionadas con la alta prevalencia de ideación suicida en una Universidad Española.

Método. Los participantes (40), con edades entre 21 y 34 años (Media = 23,90 años y Desviación Típica $=3,003$ ) fueron divididos en dos grupos según puntuación obtenida en el Inventario de Ideación Suicida de Beck (SSI) (>10 puntos) y, además, se les aplicó diversas medidas psicosociales.

Resultados. Los resultados mostraron que los estudiantes más propensos a presentar ideación suicida son menos optimistas, presentan menos habilidades sociales y un menor apoyo social percibido.

Conclusiones. La identificación precoz de los factores psicosociales relacionados con alta ideación puede ayudar en la prevención de situaciones de riesgo suicida en este colectivo.

Palabras Clave: universitarios, optimismo, habilidades sociales, apoyo social percibido, suicidio. 


\title{
High Suicidal Ideation and Psychosocial Variables in University Students
}

\begin{abstract}
Introduction. The college students have high rates of suicidal ideation often associated with psychosocial factors. The aim of this study was to evaluate whether some of these psychosocial variables are related to the high prevalence of suicidal ideation in a College Spanish.
\end{abstract}

Method. Participants $(n=40)$, aged between 21 and 34 years, Mean $=23.90$ years and Standard Deviation $=3.003$ ) were divided into two groups according to scores on the Inventory of Beck Suicide Ideation (SSI) (> 10 points) and, moreover, we applied various psychosocial measures.

Results. The results showed that students more likely to have suicidal ideation are less optimistic, have poorer social skills and less social support.

Conclusions. Early identification of psychosocial factors related to high ideation may help prevent dangerous situations in this collective suicide.

Keywords: university, optimism, social skills, perceived social support, suicide

Received: 05/15/13 Initial aceptance: 06/12/13 Final aceptance: 07/16/13 


\section{Introducción}

Existe cerca de un millón de muertes cada año en el mundo debidas al suicidio (World Health Organization-WHO, 2012a). Los jóvenes son cada vez más vulnerables a los comportamientos suicidas (WHO, 2012b, c). Los últimos datos ofrecidos en España (Instituto Nacional de Estadística - INE, 2010) informan de que un total de 425 jóvenes (349 hombres y 76 mujeres) se han suicidado entre los 20 años y los 34 años de edad, lo que supone un $13.51 \%$ sobre el total de muertes por esta causa a nivel nacional.

Entre los jóvenes, un grupo de especial vulnerabilidad suicida son los estudiantes universitarios (Mackenzie et al., 2011; O'Keefe, Tucker, Wingate y Rasmussen, 2011; Storrie, Ahern y Tuckett, 2010). Este aspecto ya había sido sugerido en varias investigaciones previas (Casullo, 1997; Kocmur y Dernovsek, 2003). Sin embargo, muy al contrario de lo que sucede en otros países (Espinoza-Gómez et al., 2010; Garlow et al., 2008; Micin y Bagladi, 2011; Skala et al., 2012), son escasos los estudios en España, en los que se valora la relación entre algunas variables y la ideación suicida en estudiantes universitarios (Ruiz-Hernández, Navarro-Ruiz, Torrente y Rodríguez, 2005). En esta línea, diversas investigaciones (Buendía, Riquelme y Ruíz, 2004, Mansilla, 2010; Nock, 2009; Pérez-Camarero, 2009) han demostrado la importancia de determinadas variables (demográficas y psicosociales), como factores moduladores del riesgo o protección para la tentativa o conducta suicida en este colectivo. En particular, respecto al género se ha sugerido que tanto la ideación suicida (Garlow et al., 2008; Rosales, Córdova y Ramos, 2012) como la incidencia de la depresión (Richardson-Vejlgaard, Sher, Oquendo, Lizardi y Stanley, 2009; Vázquez y Blanco, 2008) son más frecuentes en las universitarias que en los universitarios. También, una baja autoestima es buen un indicador de ideación suicida en universitarios (Eskin, 2012; Chioqueta y Stiles, 2007; Fergusson, Beautrais y Horwood, 2003; Villalobos-Galvis, 2009). No obstante, los estudios consultados informan de que la baja autoestima correlaciona más con la ideación que con la tentativa suicida (Fergusson, Woorward y Horwood, 2000; Marciano y Kazdin, 1994; Wilbum y Smith, 2005), fundamentalmente porque parece que son necesarias otras variables, que de forma aditiva, modulen el paso de la idea al acto en sí mismo (Fergusson et al., 2000; Orth, Trzesniewski y Robins, 2010; Rosales, Córdova y Ramos, 2012; Skala et al., 2012). 
Otras investigaciones (Ellis y Rutherford, 2008; Hirsch y Conner, 2006; Hirsch, Wolford, LaLonde, Brunk y Parker-Morris, 2009) han hallado que las inferencias de tipo cognitivo (pensamientos negativos automáticos o ideas irracionales) que realiza una persona sobre por qué le suceden acontecimientos vitales adversos (problemas familiares, económicos, académicos o de relación) es una variable moduladora en la ideación suicida. Los resultados de estos estudios informan de que los estudiantes universitarios que presentan estilos explicativos optimistas amortiguan los efectos de los acontecimientos vitales traumáticos y estresantes, reduciendo su nivel de ideación suicida; mientras que aquellos que presentan estilos explicativos pesimistas amplifican el impacto de dichos acontecimientos y contribuyen al aumento de pensamientos o conductas suicidas.

En línea con lo anterior, el optimismo disposicional hace referencia a unas expectativas estables y generalizadas de que en la vida ocurrirán cosas positivas (Scheier y Carver, 1985). El optimismo disposicional suele asociarse a una baja información de síntomas de malestar, una menor frecuencia de síntomas físicos y a un afrontamiento del estrés más óptimo (Martínez-Correa, Reyes del Paso, García-León y González, 2006; Schou, Ruland, Sandvich y Karesen, 2004). Así, recientes investigaciones (Conversano et al., 2010; Manderscheid et al., 2010) han mostrado que el optimismo disposicional es un aspecto protector contra el suicidio, que modula una menor frecuencia de ideas y tentativas suicidas.

Por otra parte, parece ser que en estudiantes universitarios, el apoyo social percibido de carácter emocional (Hirsch y Barton, 2011; Pulgar-Buendía, 2010) proveniente de estructuras sociales adaptativas (Martínez-González, Inglés, Piqueras y Ramos, 2010), es decir, de amigos y familiares sin trastornos emocionales ni tentativas de suicidio previas (Maimon y Kuhl, 2008; Winfree y Jiang, 2010) es un potente protector contra la ideación y conducta suicida (McLean, Maxwell, Platt y Harris, 2008; Meadows, Kaslow, Thompson y Jurkovic, 2005; Nakagawa et al., 2009). El hecho de que el estudiante universitario perciba que dispone de amigos y familiares que promueven actitudes positivas (de escucha, afecto y apoyo), sobre todo durante las primeras fases de ideación suicida, produce un efecto positivo directo de carácter protector contra una futura tentativa suicida (Wilcox et al., 2012). 
Además, las nuevas situaciones y relaciones con las que se enfrenta el universitario hacen que las habilidades sociales (Harris, 1995) y la conducta prosocial (Eisenberg, Fabes, y Spinrad, 2006) sean unos de los elementos clave en este periodo de la vida para alcanzar un ajuste psicosocial adecuado (Hansen, Nangle y Meyer, 1998; Inglés, Martínez-González, García-Fernández, Torregrosa y Ruiz-Esteban, 2012). Algunos autores planteaban (Durkheim, 1897; 2003; Yang y Clum, 1994) que los déficits en las destrezas y habilidades mostradas en situaciones de carácter social parecen ser buenos predictores de la tentativa suicida. En estudiantes universitarios, un alto nivel de habilidad social (Benedek, 2006; Downs y Eisenberg, 2012) promueve redes sociales de apoyo amplias, lo que produce una rápida solicitud de ayuda en las

primeras fases de gestación suicida y así, se limita el tránsito de la idea a la planificación o se minimiza la letalidad de los primeros intentos (Kleiman y Riskind, 2012).

\section{Objetivo}

Por lo tanto, el presente estudio tiene el objetivo de valorar la existencia de ideación suicida en estudiantes universitarios y comprobar qué factores psicosociales (optimismo, autoestima, apoyo social, habilidades sociales y estilos atribucionales) están asociados con una alta ideación suicida en este grupo.

\section{Método}

\section{Participantes}

El total de personas participantes $(\mathrm{N}=40)$ fueron reclutadas entre los alumnos de una asignatura de la Licenciatura en Psicopedagogía de una Universidad española (ver procedimiento). Un requisito previo para participar en la muestra fue el no haber presentado tentativas de suicidio previas, puesto que este aspecto indicaría que el participante ya ha pasado a otra fase de esta conducta. Los grupos concretos y sus características fueron:

1) El Grupo 1 sin ideación suicida ( $\mathrm{N}=21 ; 7$ hombres y 14 mujeres). Con edades comprendidas entre 21 y 33 años $(M=24.05$; DT $=2.765)$. 
2) El Grupo 2 con alta ideación suicida (N=19 personas; 5 hombres y 14 mujeres). Con edades comprendidas entre 21 y 34 años $(\mathrm{M}=23.74 ; \mathrm{DT}=$ $3.314)$.

\section{Instrumentos}

Hoja de datos sociodemográficos (confeccionada para esta investigación). En ella cada participante se reflejaron los siguientes datos: edad, sexo, estado civil (soltero, casado, separado/divorciado y viudo), situación laboral (desempleado u ocupado).

Escala de Ideación Suicida de Beck (Suicide Ideation Scale Beck et al., 1979), adaptada al castellano por Comeche, Díaz y Vallejo (1995). Esta escala cuantifica la intencionalidad suicida a través de la evaluación de los pensamientos o ideas de autoeliminación. Es una escala compuesta por 19 ítems en su versión original y 21 ítems en la versión en español. Para cada ítem hay tres alternativas de respuesta calificadas de acuerdo con su intensidad, de 0 a 2 , que indican un grado creciente de severidad y/o intensidad de la intencionalidad suicida. La suma de éstas indica la severidad de las ideas suicidas. El punto de corte (Beck et al. 1979) es una puntuación mayor o igual a 10 puntos, lo que indicaría que existe riesgo de suicidio al presentar alta ideación suicida. Diversos estudios (Beck et al., 1979; Ranieri et al., 1987) detectaron una fiabilidad alfa de Cronbach entre .83 y .89 y una alta validez de constructo (.97).

Escala de Apoyo Social (AS-25 de Pulgar-Buendía, 2010). El objetivo de esta escala es cuantificar la disponibilidad de apoyo social que tiene una persona. Consta de 25 ítems y se obtiene una puntuación total, siendo la mínima puntuación 25 y la máxima puntuación 100 puntos. La consistencia interna de la escala presenta un coeficiente alfa de Cronbach de .87 y la fiabilidad a través del procedimiento de las dos mitades presenta un coeficiente Spearman-Brown de.86.

Test de Orientación Vital (Life Orientation Test - LOT-R, de Scheier, Carver y Bridges, 1994), en la versión española de Otero, Luengo, Romero, Gómez y Castro (1998). Consta de 10 ítems que se valoran en función de una escala de 5 puntos. Esta escala valora el Optimismo Disposicional de forma unidimensional. Los autores informan de un coeficiente de fiabilidad alfa de Cronbach de $.87 \mathrm{y}$ una fiabilidad testretest de .74 . 
Escala de Autoestima (Self-Esteem Scale de Rosenberg, 1965, 1979), adaptada a población española por Vázquez, Jiménez y Vázquez (2004). Se trata de una de las escalas para la evaluación global de la autoestima. Incluye 10 ítems, cuyos contenidos se centran en los sentimientos de respeto y aceptación de sí mismo. Cada uno de los ítems se responden en una escala de cuatro puntos. La puntuación total oscila entre $10 \mathrm{y}$ 40 puntos. Según informan los autores en la traducción al castellano, su fiabilidad alfa de Cronbach es de .74 y su validez de constructo es de .87 .

Escala de Habilidades Sociales (EHS de Gismero, 2000). La escala está compuesta por 6 subescalas o factores con un total de 33 ítems. La persona debe seleccionar una de entre 4 alternativas que se le ofrecen. Una mayor puntuación global indica que la persona tiene más habilidades sociales en distintos contextos. El alfa de Cronbach de la escala completa es de .88 (Gismero, 2000).

Cuestionario de Estilo Atribucional (ASQ, de Peterson, Matousek, Mednick, Volavka Y Pollock, 1982), adaptado y traducido a población española por Segura (1983). A través de este cuestionario se evalúa el estilo atribucional de una persona (Weiner, 1985). Es un cuestionario autoaplicado que consta de 48 situaciones, donde el sujeto elige entre 2 alternativas posibles, aquella que suele utilizar habitualmente. El cuestionario se divide en 3 subescalas con 16 ítems cada una de ellas. Estas subescalas son: Estabilidad-Inestabilidad, Internalidad-Externalidad y Globalidad-Especificidad. En esta investigación se utilizó la puntuación directa global de la escala. La fiabilidad de consistencia interna medida a través del alfa de Cronbach para cada una de las dimensiones evaluadas oscila entre .72 y .75 , y la prueba presenta una validez de criterio entre .19 y .41 (Peterson et al., 1982).

\section{Procedimiento}

Se solicitó la participación de forma voluntaria a 93 alumnos de la Licenciatura en Psicopedagogía de una Universidad de España durante el mes de marzo del año 2010. Todos los alumnos eran previamente titulados universitarios en Magisterio. Posteriormente se les citó, fuera de horario lectivo, en un aula de la Universidad para la realización de las pruebas psicológicas. Junto con los cuadernillos de evaluación se les adjuntaba un consentimiento informado (Faden, Beauchamp y King, 1986) y la hoja de datos sociodemográficos. Del total de alumnos (93) de la asignatura finalmente fueron 
63 alumnos los que asistieron al lugar y hora acordados para las pruebas. Se eliminaron 3 cuadernillos, dos por estar incompletos en alguna de sus partes o faltar datos demográficos (sexo, edad, etc..) y uno por especificar que había realizado una tentativa suicida en el pasado. Se seleccionaron a todos los alumnos (20 en total) que habían obtenido una puntuación de 10 puntos o más en la Escala de Ideación Suicida de Beck et al. (1979) y se seleccionaron otros 20 alumnos de similares características sociodemográficas al grupo con ideación pero con una puntuación inferior a 10 en dicha escala. Así pues, la muestra definitiva estuvo constituida por 40 personas. Aquellas que presentaban una puntuación inferior a 10 puntos en la Escala de Ideación Suicida de Beck et al. (1979) se les denominó grupo 1, mientras que el grupo 2 estuvo constituido por aquellos universitarios que había obtenido una puntuación superior a 10 o más puntos en esta escala. El orden de aplicación de las pruebas fue contrabalanceado, utilizando para ello la técnica del contrabalanceo incompleto de cuadrado latino (Arnau, Anguera y Gómez, 1990; Buela-Casal, Caballo y Sierra, 1996), excepto el consentimiento informado y la hoja de datos sociodemográficos que siempre se administró en primer lugar y segundo lugar respectivamente. Las pruebas fueron aplicadas de forma colectiva.

\section{Resultados}

Para analizar la existencia de diferencias en las distintas variables medidas entre los dos grupos se utilizó el análisis de $t$ Student. En aquellos casos en los que no se cumplían los supuestos de normalidad y homocedasticidad se usó la prueba no paramétrica U de Mann-Withney. El nivel de significación estadística requerido en todas las pruebas fue de $p<0,05$. El análisis estadístico de los datos se realizó mediante el paquete estadístico SPSS versión 19.0.

Para medir el efecto de las variables extrañas (sexo, edad, estado civil, etc.) se calculó la razón de verosimilitud entre dichas variables y la variable grupo. El análisis de los datos obtenidos informa de que los grupos son homogéneos en todas las variables: Sexo $\left(\mathrm{RV}_{(1)}=.235 ; p=.628\right)$; edad $\left(F_{1,40}=.104 ; p=.748\right)$, situación laboral $\left(\mathrm{RV}_{(1)}=.405 ; p=.524\right)$, o estado civil $\left(\mathrm{RV}_{(2)}=.374 ; p=.829\right)$.

Los resultados (Tabla 1) referidos al nivel de apoyo social han mostrado que existen diferencias significativas en las puntuaciones medias en esta variable entre los 
grupos $\left(t_{40}=10.500 ; p<.001\right)$, así existe un mayor nivel de apoyo en aquellos participantes sin ideas de suicidio respecto a aquellos con ideas de suicidio. De igual forma, existen diferencias significativas entre los grupos en cuanto a su nivel de optimismo $\left(t_{40}=14.361 ; p<.001\right)$, lo que muestra un menor nivel de optimismo disposicional en el grupo con ideas de suicidio respecto al grupo que no presenta ideas de suicidio. También se obtuvo una diferencia significativa respecto al nivel de autoestima $(\mathrm{U}=14.500 ; p<.001)$, así, como en el nivel de habilidades sociales $\left(t_{40}=\right.$ 9.783; $p$ <.001). Lo que informa de un mayor nivel de autoestima y habilidades sociales en aquellos estudiantes sin ideas de suicidio respecto a aquellos que tienen ideas de suicidio. Ver Tabla 1.

Tabla 1. Valores descriptivos y nivel de significación de las variables psicológicas por grupos

\begin{tabular}{cccccccc}
\hline \multirow{2}{*}{ Prueba (variable) } & \multicolumn{2}{c}{ grupo 1 } & \multicolumn{2}{c}{ grupo 2 } & \multirow{2}{*}{ (con ideación) } & \multirow{2}{*}{$T_{40}$} & \multirow{2}{*}{$p<.05$} \\
\cline { 2 - 5 } & Media & DT & Media & DT & & \\
\hline AS-25 (apoyo social) & 87.86 & 15.48 & 26.74 & 7.78 & 10.50 & $.00^{*}$ \\
LOT-R (optimismo) & 33.95 & 5.67 & 13.11 & 2.92 & 14.36 & $.00^{*}$ \\
S-ES (autoestima) & 41.76 & 10.39 & 14.37 & 2.91 & 14.50 & $.00^{* *}$ \\
EHS (habilidades sociales) & 89.29 & 22.27 & 27.53 & 16.96 & 9.78 & $.00^{*}$ \\
ASQ (estilo atribucional) & 1.10 & 4.85 & .26 & 3.36 & .62 & .53 \\
\hline$* t$ Student $\left(t_{40} ; p<.01\right)$ & $* *$ Estadístico no paramétrico de U de Mann-Whitney $(p<.01)$ &
\end{tabular}

\section{Discusión}

Se exploró la existencia de ideación suicida en estudiantes universitarios, con el objetivo de comprobar qué factores psicosociales (optimismo, estilo atribucional, autoestima, apoyo social y habilidades sociales) confieren una mayor vulnerabilidad suicida asociada a las primeras fases de esta conducta.

Al igual que investigaciones previas (Espinoza-Gómez et al., 2010; Garlow et al., 2008; González-Forteza, García, Medina-Mora y Sánchez, 1998; Sanz, Navarro y Vázquez, 2003; Vázquez y Blanco, 2006) los resultados obtenidos indicaron que la ideación suicida presenta una alta prevalencia (33.3\% sobre el total) en los estudiantes universitarios de este estudio. Pero además, los alumnos con alta ideación suicida puntúan significativamente más bajo en autoestima, habilidades sociales, apoyo social y optimismo disposicional que los alumnos con baja ideación suicida, en línea con otras 
investigaciones (Córdova, Rosales, Caballero y Rosales, 2007; Eskin, 2012; VillalobosGalvis, 2009; Skala et al., 2012).

A este respecto, el hecho de que culturalmente se exija y se valora cada más la responsabilidad de los actos personales (Farabaugh et al., 2012; Vázquez Y Blanco, 2008), y en particular el éxito académico (Rosales, Córdova y Ramos, 2012; Inglés et al., 2009; Villalobos-Galvis, 2009), podría hacer que en universitarios la autoestima y el optimismo asuman un valor importante en la predicción de las ideas suicidas, pues un fracaso (o su posible anticipación) en alguna de las áreas de su vida, afectará directamente a la imagen que tienen de sí mismos, disminuyendo con ello su autovalía y haciendo más probable la presencia de ideas suicidas. Tal y como lo plantean otros autores (Arria et al., 2009) se considera imprescindible la puesta en marcha de intervenciones psicológicas tempranas, basadas en el desarrollo de programas de fomento del optimismo y la focalización en las propias capacidades, como fórmula para asumir pequeños fracasos como paso previo hacia el éxito académico en el ámbito universitario.

En la línea con estudios previos (Joiner el al., 2009; McLean et al., 2008; Nakagawa et al., 2009; Walker, Wingate, Obasi y Joiner, 2008) los resultados obtenidos en esta investigación sobre los mayores niveles de apoyo social emocional percibido en los estudiantes sin ideas de suicidio en comparación con los que tienen ideas de suicidio, demuestran la importancia de las redes sociales adaptativas (Brougham, Zail, Mendoza y Miller, 2009; Luxton, June y Fairall, 2012; Martínez-González et al. 2010; Matlin, Molock y Tebes, 2011) como herramientas eficaces de promoción de la salud en este colectivo. Una interpretación de este hallazgo podría ser porque aquellos estudiantes con mayores niveles de apoyo social percibido ponen en marcha habilidades sociales más adecuadas, creando vínculos emocionales más cercanos con compañeros de clase y amigos. Estas redes sociales actuarían como alarmas de detección eficaces, sobre todo en las primeras fases de la conducta suicida (ideación). Lo que hace necesario la puesta en marcha y reforzamiento de instrumentos institucionalizados dentro de los dispositivos de salud del campus donde se reflejen aquellos miembros de las redes sociales sustitutivas como apoyo social clave en universitarios en riesgo o con antecedentes de alteraciones psicológicas. Así, el desarrollo de campañas de sensibilización entre el colectivo universitario para la 
detección precoz de problemas de salud mental entre compañeros podría eliminar la estigmatización social vinculada a la solicitud de ayuda en las fases iniciales de malestar y promover redes de apoyo social reglado dentro de las propias asociaciones universitarias o a través de herramientas institucionalizadas de carácter on-line en el propio campus.

\section{Limitaciones y perspectivas futuras}

En primer lugar, la muestra es muy pequeña y compuesta en exclusividad por estudiantes de psicopedagogía, por lo que los resultados pueden no ser generalizables ni a estudiantes de psicopedagogía ni a universitarios de otras titulaciones. Sería de interés valorar la ideación suicida en muestras más grandes y que pudieran incluir estudiantes de otras titulaciones universitarias, además de realizar otro tipo de análisis (p.e.: análisis de regresión logística). En segundo lugar, la muestra era eminentemente femenina, lo que puede haber aumentado la probabilidad de incrementar los niveles de ideación suicida (González-Forteza et al., 1998; Rosales, Córdova y Ramos, 2012; Sánchez, Cáceres Y Gómez, 2002). Aunque es cierto que en Humanidades y Ciencias de la Educación, a la que pertenece la titulación de Psicopedagogía, existe siempre una amplia mayoría de mujeres, sería importante para futuros investigadores, recopilar datos suficientes para obtener tamaños de muestra similares cuando se comparan los factores de riesgo para el suicidio entre grupos de universitarios. Finalmente decir, que existen otros factores de riesgo pertinentes para la evaluación de la ideación suicida que no han sido valorados en este estudio. Investigaciones futuras deben considerar los factores socioeconómicos, familiares, culturales, educativos y epigenéticos, que muchas investigaciones (Amitai y Apter, 2012; Brent y Melhem, 2008; International Association for Suicide Prevention-IASP, 2010; Joe, Canetto y Romer, 2008; Joiner, 2006; Programa de las Naciones Unidas para el Desarrollo-PNUD, 2009; SánchezTeruel, 2012; Wasserman, Terenius, Wasserman y Sokolowski, 2010; WHO, 2012 a, b, c) ya han demostrado que están asociados a la ideación y riesgo de suicidio.

En particular, estudios posteriores sobre ideación suicida en universitarios deberían incluir medidas de detección temprana de la ansiedad (estado-rasgo), de la depresión y desesperanza, personalidad y valorar los posibles acontecimientos adversos anteriores (tener un familiar enfermo, presentar una enfermedad crónica, si existen antecedentes de trastornos psicológicos, etc.). Al igual que ya plantean otros autores 
(Bausela, 2005) las universidades españolas precisan de un servicio de evaluación y tratamiento psicológico más homogéneo, de carácter nacional y guiado por profesionales de la psicología a tiempo completo. Del tal manera, que se pudieran implementar por estos profesionales programas de prevención, evaluación y tratamiento gratuito a estudiantes universitarios en el propio campus.

\section{Referencias}

Amitai, M. y Apter, A. (2012). Social aspects of suicidal behavior and prevention in early life: a review. International Journal of Environmental Research and Public Health, 9 (3), 985-994. doi: http://dx.doi.org/10.3390/ijerph9030985

Arnau, J. Anguera, M.T. y Gómez, J. (1990). Metodología de la investigación en ciencias del comportamiento. Murcia: Universidad de Murcia. Secretariado de publicaciones

Arria, A.M., O’Grady, K.E.N., Caldeira, K.M., Vincent, K.B., Wilcox, H.C. y Wish, E.D. (2009). Suicide ideation among college students: A multivariate analysis. Archives of Suicide Research, 13 (3), 230-246. doi: http://dx.doi.org10.1080/13811110903044351

Bausela, E. (2005). Diseño de un servicio de orientación psicológica en el contexto de la Universidad de León. Revista de Psicodidáctica, 10, (2), 123-140. doi: http://dx.doi.org/10.1387/RevPsicodidact.194

Beck, A.T., Kovacs, M. y Weissman, A. (1979). Assessment of suicidal intention: the scale for suicide ideation. Journal Counseling Clinical Psychology, 41, (2), 343352.

Benedeck, E.P. (2006). Suicidal behavior in Europe: results from the Who/Euro multicentre study on suicidal behavior. Bulletin of the Menninger Clinic, 70, 245-248.

Brent, A.A. y Melhem, N. (2008). Familial transmission of suicidal behaviour. Psychiatric Clinics of North America, 31 (2), 157-177. doi: http://dx.doi.org/10.1016/j.psc.2008.02.001

Brougham, R. R., Zail, C. M., Mendoza, C. M., y Miller, J. R. (2009). Stress, sex differences and coping strategies among college students. Current Psychology, 28 (2), 85-97. doi: http://dx.doi.org/10.1007/s12144-009-9047-0 
Buela-Casal, G., Caballo, V. y Sierra, J. C. (1996). Manual de evaluación en psicología clínica y de la salud. Madrid: Siglo XXI.

Buendía, J., Riquelme, A. y Ruíz, J.A. (2004). El suicidio en adolescentes. Murcia. Universidad de Murcia.

Casullo, M.M. (1997). Riesgo suicida en estudiantes universitarios: Epidemiología y prevención. Desarrollos en Psiquiatría Argentina, 2, 33-38.

Chioqueta, A.P. y Stiles, T.C. (2007). Dimensions of the dysfunctional attitude scale (das-a) and the automatic thoughts questionnaire (atq-30) as cognitive vulnerability factors in the development of suicide ideation. Behavioural and Cognitive Psychotherapy, 35 (5), 579-589. doi: http://dx.doi.org/10.1017/S1352465807003803

Comeche, M. I., Díaz, M. y Vallejo, M. (1995).Cuestionarios, inventarios y escalas. Ansiedad, depresión y habilidades sociales. Madrid: Universidad-Empresa.

Conversano, C., Rotondo, A., Lensi, E., Della Vista, O., Arpone, F. y Reda, M.A. (2010). Optimism and its impact on mental and physical well-being. Clinical Practice and Epidemiology in Mental Health, 14, 25-29. doi: http://dx.doi.org/10.2174/1745017901006010025

Córdova, M., Rosales, M.P., Caballero, R. y Rosales, J.C. (2007). Ideación suicida en jóvenes universitarios: $\mathrm{Su}$ asociación con diversos aspectos psicosociodemográficos. Psicología Iberoamericana 15 (2), 17-21

Dua, J.K. (1994). Comparative predictive value of attribution style, negative affect, and positive affect in predicting self-reported physical health and psychological health. Journal of Psychosomatic Research, 38 (7), 669-680. doi: http://dx.doi.org/10.1016/0022-3999(94)90019-1

Durkheim, E. (1897). Le suicide. Paris: Alcain.

Durkheim, E. (2003). El suicidio. Buenos Aires: Losada (orig. 1897)

Downs, M.F. y Eisenberg, D. (2012). Help seeking and treatment use among suicidal college students. Journal of American College Health, 60, 104-114. doi: http://dx.doi.org/10.1080/07448481.2011.619611

Elliott, J.L. y Frude, N. (2001). Stress, coping styles, and hopelessness in self-poisoners. Journal of Crisis Intervention and Suicide Prevention, 22 (2), 20-26. doi: http://dx.doi.org/10.1027//0227-5910.22.1.20 
Ellis, T.E. y Rutherford, B. (2008). Cognition and suicide: two decades of progress. International Journal of Cognitive Therapy, 1 (1), 47-68. doi: http://dx.doi.org/10.1680/ijct.2008.1.1.47

Espinoza-Gómez, F., Zepeda-Pamplona, V., Bautista-Hernández, V., HernándezSuárez, C.M., Newton-Sánchez, O.A. y Plasencia-García, G.R. (2010). Violencia doméstica y riesgo de conducta suicida en universitarios adolescentes. Revista de Salud Pública Mexicana, 52(3), 213-219

Eskin, M. (2012). The Role of childhood sexual abuse, childhood gender nonconformity, self-esteem and parental attachment in predicting suicide ideation and attempts in Turkish young adults. Suicidology Online,3, 114-123

Faden, R., Beauchamp, T. y King, N. (1986). A history and theory of informed consent. New York: Oxford University Press

Farabaugh, A., Bitran, S., Nyer, M., Holt, D.J., Pedrelli, P., Shyu, I., ...y Fava, M. (2012). Depression and suicidal ideation in college students. International journal of Experimental Psychopathology, Phenomenology and Psychiatric Diagnosis, 45 (4), 228-234. doi: http://dx.doi.org/10.1159/000331598

Fergusson, D.M., Woorward, L.J. y Horwood, L.J. (2000). Risk factors and life processes associated with the onset of suicidal behaviour during adolescence and early adulthood. Psychological Medicine, 30(1), 23-39. doi: http://dx.doi.org/10.1017/S003329179900135X

Fergusson, D. M., Beautrais, A. L. y Horwood, L. J. (2003). Vulnerability and resiliency to suicidal behaviors in young people. Psychological Medicine, 33(1), 61-73. doi: http://dx.doi.org/10.1017/S0033291702006748

Fernández-Prieto, M. (2001). Análisis comparativo entre el estilo atribucional y la autoestima en pacientes delirantes y depresivos. Tesis doctoral no publicada. Universidad de Granada

Gismero, E. (2000). EHS: Escala de habilidades sociales. Madrid: Tea ediciones

Garlow, S.J., Rosenberg, J., Moore, J.D., Hass, A.P., Koestner, B., Hendin, H. y Nemeroff, Ch.B. (2008). Depression, desperation, and suicidal ideation in college students: Results from the American Foundation for Suicide Prevention College Screening Project at Emory University. Depression and Anxiety, 25(6), $482-488$ 
González-Forteza, C., García G., Medina-Mora, E. y Sánchez, M. A. (1998). Indicadores psicosociales predictores de ideación suicida en dos generaciones de estudiantes universitarios. Salud Mental, 21(3), 3,1-8

Hansen, D.J., Nangle, D.W. y Meyer, K.A. (1998). Enhancing the effectiveness of social skillsinterventions with adolescents. Education and Treatment of Children, 21(4), 489-513

Harris, J. R. (1995). Where is the child's environment?: A group socialization theory of development. Psychological Review, 102(3), 458-489. doi: http://dx.doi.org/10.1037//0033-295X.102.3.458

Hawkins, M.T. y Miller, R.J. (2003). Cognitive vulnerability and resilience to depressed mood. Australian Journal of Psychology, 55(3), 176-183. doi: http://dx.doi.org/10.1080/0004953042000298642

Hirsch, J.K. y Conner, K.R. (2006). Dispositional and explanatory style optimism as potential moderators of the relationship between hopelessness and suicidal ideation. Suicide and Life-Threatening Behavior, 36(6), 661-669. doi: http://dx.doi.org/10.1521/suli.2006.36.6.661

Hirsch, J.K., Wolford, K., Lalonde, S.M., Brunk, L. y Parker-Morris, A. (2009). Optimistic explanatory style as a moderator of the association between negative life events and suicide ideation. The journal of Crisis Intervention and Suicide Prevention, 30(1), 48-53. doi: http://dx.doi.org/10.1027/0227-5910.30.1.48

Hirsch, J.K. y Barton, A.L. (2011). Positive social support, negative social exchanges, and suicidal behavior in college students. Journal of American College Health, 59(5,) 393-398. doi: http://dx.doi.org/10.1080/07448481.2010.515635

Inglés, C. J., Benavides, G., Redondo, J., García-Fernández, J. M., Ruiz-Esteban, C., Estévez, C., y Huescar, E. (2009). Conducta prosocial y rendimiento académico en estudiantes españoles de Educación Secundaria Obligatoria. Anales de Psicología, 25(1), 93-101

Inglés, C.J., Martínez-González, A.E., García-Fernández, J.M., Torregrosa, M. y RuizEsteban, C. (2012). Conducta prosocial y autoconcepto en una muestra de estudiantes españoles de educación secundaria obligatoria. Revista de Psicodidáctica, 17(1), 135-156. doi: http://dx.doi.org/10.1387/RevPsicodidact.1861

Instituto Nacional de Estadística-INE (2010). Tasas de mortalidad en España. Madrid: INE 
International Association for Suicide Prevention-IASP (2010). Día mundial de la prevención del suicidio-10 de septiembre de 2010. Recuperado de http://www.iasp.info/

Joe, S., Canetto, S.S. y Romer, D. (2008). Advancing prevention research on the role of culture in suicide prevention. Suicide and Life-Threatening Behavior, 38(3), 354-362. doi: http://dx.doi.org/10.1521/suli.2008.38.3.354

Joiner, T. (2006). Why people die by suicide?. Boston: Harvard university press

Joiner, T. E., Van Orden, K., Witte, T. K., Selby, E. A., Ribeiro, J. D., Lewis, R., . . y Rudd, M. D. (2009). Main predictions of the interpersonal-psychological theory of suicidal behavior: empirical tests in two samples of young adults. Journal of Abnormal Psychology, 118(3), 634-646. doi: http://dx.doi.org/10.1037/a0016500

Kocmur, M. y Dernovsek, M. Z. (2003). Attitudes towards suicide in Slovenia: a crosssectional survey. International Journal of Social Psychiatry, 49(1), 8-16. doi: http://dx.doi.org/10.1177/0020764003049001537

Kleiman, E.M. y Riskind, J. H. (2012). Utilized social support and self-esteem mediate the relationship between perceived social support and suicide ideation. Crisis: The Journal of Crisis Intervention and Suicide Prevention, 12(1), 1-8. doi: http://dx.doi.org/10.1027/0227-5910/a000159

Luxton, D.D., June, J.D. Y Fairall, J.M. (2012). Social media and suicide: a public health perspective. American Journal of Public Health, 102(2), 195-200. doi: http://dx.doi.org/10.2105/AJPH.2011.300608

Mackenzie, S., WiegeL, J.R., Mundt, M., Brown, D., Saewyc, E., Heiligenstein, E. y Fleming, M. (2011). Depression and suicide ideation among students accessing campus health care. American Journal of Orthopsychiatry, 81(1), 101-107. doi: http://dx.doi.org/10.1111/j.1939-0025.2010.01077.x

Manderscheid, R.W, Ryff, C.D., Freeman, E.J., Mcknight-Eily, L.R, Dhingra, S. y Strine, T.W. (2010). Evolving definitions of mental illness and wellness. Preventing chronic disease, 7(1), 1-6 Available in http://www.cdc.gov/pcd/issues/2010/jan/09_0124.htm.

Mansilla, F. (2010). Suicidio y prevención. Palma de Mallorca: Intersalud

Marciano, P. y Kazdin, E. (1994). Self-esteem, depression, hopelessnes and suicidal intent among psychiatrically disturbed inpatient children. Journal of Clinical 
Child Psychology, 23(2), 151-160. doi: http://dx.doi.org/10.1207/s15374424jecp2302_5

Martínez-Correa, A., Reyes del Paso, G.A., García-León, A. y González, M.I. (2006). Análisis de la relación entre el optimismo/pesimismo disposicional y las estrategias de afrontamiento del estrés en la predicción de la sintomatología somática autoinformada. Psicothema, 18, 66-72

Martínez-González, A. E., Inglés, J.C., Piqueras, J.A. y Ramos, V. (2010). Importancia de los amigos y los padres en la salud y el rendimiento académico. Electronic Journal of Research in Educational Psychology, 20(1), 111-138

Maimon, D. y Khul, D. C. (2008). Social control and youth suicidality: situating durkheim's ideas in a multilevel framework. American Sociological Review, 73(6), 921-943. doi: http://dx.doi.org/10.1177/000312240807300603

Matlin, S. L., Molock, S. D. y Tebes, J. K. (2011). Suicidality and depression among africanamerican adolescents: the role of family and peer support and community connectedness. American Journal of Orthopsychiatry, 81(1), 108-117. doi: http://dx.doi.org/10.1111/j.1939-0025.2010.01078.x

Mclean, J., Maxwell, M., Platt, S. y Harris, F. (2008). Risk and protective factors for suicide and suicidal behavior: a literature review. Edinburgh: Scottish Government Social Research

Meadows, L.A., Kaslow, N.J., Thompson, M.P. y Jurkovic, G.J. (2005). Protective factors against suicide attempt risk among african-american women experiencing intimate partner violence. American Journal of Community Psychology, 36(1-2), 109-121. doi: http://dx.doi.org/10.1007/s10464-005-6236-3

Micin, S. y Bagladi, V. (2011). Salud Mental en estudiantes universitarios: Incidencia de psicopatología y antecedentes de conducta suicida en población que acude a un servicio de salud estudiantil. Terapia Psicológica, 29(1), 53-64. doi: http://dx.doi.org/10.4067/S0718-48082011000100006

Nakagawa, M., Kawanishi, C., Yamada, T., Iwamoto, Y., Sato, R., Hasegawa, H., y Hirayasu, Y. (2009). Characteristics of suicide attempters with family history of suicide attempt: a retrospective chart review. BMC Psychiatry, 9, 32-38. doi: http://dx.doi.org/10.1186/1471-244X-9-32

Nock, M.K. (2009). Suicidal behavior among adolescents: correlates, confounds, and (the search for) causal mechanisms. Journal of the American Academy of Child $y$ 
Adolescent Psychiatry, 48, 237-239. doi: http://dx.doi.org/10.1097/CHI.0b013e318196b944

Orth, U., Trzesniewski, K.H. y Robins, R.W. (2010). Self-esteem development from young adulthood to old age: a cohort-sequential longitudinal study. Journal of Personality and Social Psychology, 98(4), 645-658. doi: http://dx.doi.org/10.1037/a0018769

O'keefe, V. M., Tucker, R. P., Wingate, L.R. y Rasmussen, K. A. (2011). American Indian hope: a potential protective factor against suicidal ideation. Journal of Indigenous Research, $1, \quad$ article $3 . \quad$ Available in http://digitalcommons.usu.edu/kicjir/vol1/iss2/3

Otero, J.M., Luengo, A., Romero, E. Gómez, J.A. y Castro, C. (1998). Psicología de personalidad. Manual de prácticas. Barcelona: Ariel Practicum.

Pérez-Camarero, S. (2009). El suicidio adolescente y juvenil en España. Revista de Estudios de Juventud, 84, 126-142

Peterson K.G, Matousek, M., Mednick, S.A., Volavka, J. y Pollock V. (1982). Age antecedents of thievery. Acta Psychiatrica Scandinavica, 65(5), 331-338. doi: http://dx.doi.org/10.1111/j.1600-0447.1982.tb00854.x

Programa de las Naciones Unidas para el Desarrollo (PNUD) (2009). Informe sobre desarrollo humano 2009. Superando barreras: movilidad y desarrollo humanos. Recuperado de http://hdr.undp.org/en/media/hdr_2009_es_indicators.pdf

Pulgar-Buendía, A. (2010). Aplicación del modelo biopsicosocial para la comprensión de la calidad de vida y el pronóstico en el cáncer hematológico. Tesis doctoral no publicada. Universidad de Jaén.

Ranieri, WF, Steer RA, Kovacs M, Lavrence, T. I., Rissmiller, D. J., Piper, G. E., y Beck, A. T. (1987). Relationships of depression, hopelessness, and dysfunctional attitudes to suicide ideation in psychiatric patients. Psychological Reports, 61(3), 967-975. doi: http://dx.doi.org/10.2466/pr0.1987.61.3.967

Richardson-Vejlgaard, R., Sher, L., Oquendo, M.A., Lizardi, D. y Stanley, B. (2009). Moral objections to suicide and suicidal ideation among mood disordered Whites, Blacks, and Hispanics. Journal of Psychiatric Research, 43(4), 360365. doi: http://dx.doi.org/10.1016/j.jpsychires.2008.03.008

Rosales, J.C., Córdova, M. y Ramos, R. (2012). Ideación suicida en estudiantes mexicanos: un modelo de relación múltiple con variables de identificación personal. Psicología y Salud, 22(1), 63-74 
Rosenberg, M. (1965). Society and adolescent self-image. New Jersey: Princeton University press.

Rosenberg, M. (1979). Conceiving the self. New York: Basic Books.

Ruiz-Hernández, J.A., Navarro-Ruiz, J.M., Torrente, G. y Rodríguez, A. (2005). Construcción de un cuestionario de creencias actitudinales sobre el comportamiento suicida: el CCCS-18. Psicothema 17, 684-690

Sánchez-Teruel, D. (2012). Variables sociodemográficas y biopsicosociales relacionadas con la conducta suicida. En J. A. Muela, A. García y A. Medina (eds.). Perspectivas en psicología aplicada (pp. 61-78). Jaén: Centro Asociado Andrés de Vandelvira de la U.N.E.D.

Sánchez, R., Cáceres, H. y Gómez, D. (2002). Ideación suicida en adolescentes universitarios: prevalencia y factores asociados. Biomédica, 22(su2), 407-416

Sanz, J. Navarro, M.E. y Vázquez, C. (2003). Adaptación española para el inventario de la depresión de Beck-II (BDI-II): propiedades psicométricas en estudiantes universitarios. Análisis y Modificación de Conducta, 29(124), 239-288

Scheier, M.F. y Carver, C.S. (1985). Optimism, coping and health: assessment and implication of generalized outcome expectancies. Health Psychology, 4(3), 219247. doi: http://dx.doi.org/10.1037//0278-6133.4.3.219

Scheier, M.F., Carver, C.S. y Bridges, M.W. (1994). Distinguising optimism from neuroticism (and trait anxiety, self-mastery and self-esteem): A reevaluation of the life orientation test. Journal of Personality and Social Psychology, 67(6), 1063-1078. doi: http://dx.doi.org/10.1037//0022-3514.67.6.1063

Scheier, M. F., Carver, C. S., y Bridges, M. W. (2001). Optimism, pessimism, and psychological well-being. In e. C. Chang (ed.), optimism and pessimism: implications for theory, research, and practice (pp. 189-216). Washington, DC: American Psychological Association.

Segura, M. (1983). Cuestionario de Estilo Atribucional. Segundas Jornadas de Modificación de Conducta. Madrid: Centro de Investigación y Terapia de Conducta.

Skala, K., Kapusta, N.D., Schlaff, G., Unseld, M., Erfurth, A., Lesch, O.M., Walter, H., Akiskal, K.K. y Akiskal, H.S. (2012). Suicidal ideation and temperament: An investigation among college students. Journal of Affective Disorders, 141(2-3), 399-405. doi: http://dx.doi.org/10.1016/j.jad.2012.03.010 
Storrie, K., Ahern, K. y Tuckett, A. (2010). A systematic review: students with mental health problems - a growing problem. International Journal of Nursing Practice, 16(1), 1-6. doi: http://dx.doi.org/10.1111/j.1440-172X.2009.01813.X

Schou, I., Ruland, C.M., Sandvik, L. y Karesen, R. (2004). Pessimism as a predictor of emotional morbidity one year following breast cancer surgery. Psychooncology, 13(5), 309-320. doi: http://dx.doi.org/10.1002/pon.747

Vázquez, F.L. y Blanco, V. (2006). Symptoms of depression and related factors among spanish university students. Psychological Reports, 99(2), 583-590. doi: http://dx.doi.org/10.2466/PR0.99.6.583-590

Vázquez, F.L. y Blanco, V. (2008). Prevalence of DSM-IV major depression among spanish university students. The Journal of American College Health, 57(2), 165-171. doi: http://dx.doi.org/10.3200/jach.57.2.165-172

Vázquez, A. y Manassero, M.A. (1989). La teoría de la atribución y el rendimiento escolar. Educació i cultura: revista mallorquina de pedagogía, 7, 225-241.

Vázquez, J.A., Jiménez, R. y Vázquez, R. (2004). Escala de autoestima de Rosenberg. Apuntes de Psicología 22, 247-255.

Villalobos-Galvis, F.H. (2009). Ideación suicida en jóvenes: formulación y validación del modelo integrador explicativo en estudiantes de educación secundaria y superior. Tesis doctoral. Universidad de Granada

Walker, R. L., Wingate, L. R., Obasi, E. M., y Joiner, T. (2008). An empirical investigation of acculturative stress and ethnic identity as moderators for depression and suicidal ideation in college students. Cultural Diversity and Ethnic Minority Psychology, 14(1), 75-82. doi: http://dx.doi.org/10.1037/1099$\underline{9809.14 .1 .75}$

Wasserman, D., Terenius, L., Wasserman, J. y Sokolowski, M. (2010). The 2009 Nobel conference on the role of genetics in promoting suicide prevention and the mental health of the population. Molecular Psychiatry, 15(1), 12-17. doi: http://dx.doi.org/10.1038/mp.2009.113

Weiner, B. (1985). An attributional theory of achievement motivation and emotion. Psychological Review, 92(4), 548-573. doi: http://dx.doi.org/10.1037//0033295X.92.4.548

Wilbum, V.R. y Smith, D.E. (2005). Stress, self-esteem and suicidal ideation in late adolescents. The Journal of Adolescence, 40(157), 33-45. 
Wilcox, H.C., Arria, A.M., Caldeira, K.M., Vincent, K.B., Pinchevsky, G.M. y O'grady, K.E. (2012). Longitudinal predictors of past-year non-suicidal self-injury and motives among college students. Psychological Medicine, 42(4), 717-26. doi: http://dx.doi.org/10.1017/s0033291711001814

Winfree, L.T. y Jiang, S. (2010). Youthful suicide and social support exploring the social dynamics of suicide-related behaviour and attitudes within a national sample of U.S. adolescents. Youth Violence and Juvenile Justice, 8(1), 19-37. doi: http://dx.doi.org/10.1177/1541204009338252

World Health Organization-WHO (2012a).Suicide prevention (supre).Recuperado de http://www.who.int/mental_health/prevention/en/

World Health Organization-WHO (2012b).Suicide prevention (supre). Recuperado de http://www.who.int/mental_health/prevention/suicide/suicideprevent/en/index.ht $\mathrm{ml}$

World Health Organization-WHO (2012c). Public health action for the prevention of suicide: a framework. Geneva: who document production services

Yang, B. y Clum, G.A. (1994). Life stress, social support, and problem-solving skills predictive of depressive symptoms, hopelessness and suicide ideation in an Asian student population: a test of a model. Suicide and Life-Threatening Behavior, 24(2), 127-139 